\title{
Large Power Swings in Doped-Fiber Amplifiers with Highly Variable Data
}

\author{
A. Bononi, Member, IEEE, L. Tančevski, Member, IEEE, and L. A. Rusch, Member, IEEE
}

\begin{abstract}
We show that doped-fiber amplifiers operated in saturation can display very fast transient dynamics and large power swings in a packet switching environment with highly variable packet interarrival times.
\end{abstract}

Index Terms-EDFA, optical networks, self-similar traffic.

\section{INTRODUCTION}

$\mathbf{T}$ HERE IS growing interest in the transient response of erbium-doped fiber amplifiers (EDFA's) to channel gain or loss in wavelength-division-multiplexing (WDM) circuit switching networks. Infrequent and abrupt power changes at the EDFA input, which due to the network reconfiguration time occur on a time scale equal to or greater than a millisecond, allow enough time for the EDFA to reach substantially different steady-state values. In WDM slotted packet switching networks, where no power is transmitted on empty slots, the abrupt power fluctuations due to random packet arrivals at the input of EDFA are much more frequent, but may still cause very fast power transients and large output power swings. This happens when the input packet power is large and the interarrival times have large variability, such as with selfsimilar multimedia traffic [2].

In this letter, we examine the influence of the statistics of the packet interarrival times on the distribution of the EDFA output power in a 16-channel WDM system feeding a single EDFA. The input packetized traffic is modeled as a superposition of 16 independent ON/OFF asynchronous transfer mode (ATM) slotted sources, with the duration of the ON and OFF times, in slots, taken from a (rounded) Pareto distribution. It is known that the superposition of many ON/OFF sources whose ON and OFF times have infinite variance produces selfsimilar traffic [2].

We present simulations where the variance of the Pareto ON/OFF distribution gradually approaches infinite values. Large power swings are found at the output of the EDFA in the selfsimilar (infinite variance) case, indicating potential nonlinear optical distortions and receiver overload problems in networks with highly variable multimedia packetized traffic.

Manuscript received June 19, 1998; revised September 11, 1998. This work was supported by the Italian National Research Council under Grant CNR 97.00388.CT12, and under a research grant from QuébecTel.

A. Bononi is with Università di Parma, Dipartimento di Ingegneria dell'Informazione, I-43100 Parma, Italy.

L. Tančevski is with Alcatel Network Systems, Richardson, TX 75081 USA.

L. A. Rusch is with the Département de génie électrique et de génie informatique, Université Laval, Ste-Foy, PQ, G1K-7P4 Canada.

Publisher Item Identifier S 1041-1135(99)00361-4.

\section{Simulation Model}

The system under study is composed of an EDFA fed by 16 WDM time-slotted channels, with signals at wavelengths 1544-1559 nm, with 1-nm uniform spacing. The channels carry 53-byte ATM cells at a bit rate of $150 \mathrm{Mb} / \mathrm{s}(2.82 \mu \mathrm{s}$ slot duration) and peak input power per channel of $-3 \mathrm{dBm}$. The amplifier spectral parameters (absorption coefficients and saturation powers) were taken from [3, Table 4.2]; in order to obtain the full spectra from the fitted parameters we use the Lorentizian formula, i.e.,

$$
\sigma_{a}=\sigma_{a}^{\text {peak }} * \sum_{i=1}^{8} a_{i} *\left[1+4\left(\omega-\omega_{i}\right)^{2} / \Delta \omega_{i}^{2}\right]^{-1} .
$$

Forward pumping at $980 \mathrm{~nm}$ with pump power $18.4 \mathrm{dBm}$ was assumed. Other EDFA parameters: fluorescence time $\tau=10.5$ ms and length $35 \mathrm{~m}$, providing 34-dB small-signal gain.

For each independent ON/OFF ATM source, the ON times $T_{\mathrm{on}}$ were simulated as

$$
T_{\text {on }}=\left\lfloor\frac{1}{U^{1 / \alpha_{\text {on }}}}\right\rfloor
$$

where $U$ is a random variable uniformly distributed on $[0,1]$ and $\lfloor x\rfloor$ indicates the floor function. $T_{\text {off }}$ was generated similarly. $T_{\mathrm{on}}$ has a rounded Pareto distribution, variance is infinite when $1 \leq \alpha_{\mathrm{on}} \leq 2$ [2]. Channel utilization is given as $\rho=E[\mathrm{ON}] /(E[\mathrm{ON}]+E[\mathrm{OFF}])$. Specifying $\alpha_{\mathrm{on}}$ and $\rho$, gives

$$
\alpha_{\text {off }}=\frac{(1-\rho) \alpha_{\mathrm{on}}}{(1-\rho) \alpha_{\mathrm{on}}-\rho\left(\alpha_{\mathrm{on}}-1\right)} .
$$

The $\alpha$-parameters were used to simulate traffic with different variability of ON/OFF times, ranging from relatively smooth traffic $(\alpha \gg 2)$ to highly bursty traffic $(1<\alpha<2)$. Highly bursty traffic entails a large probability of long lulls between cells, which give sufficient time for the EDFA gain to recover its small-signal value, thus causing high-power levels and gain sag across the cells following the lulls. In order to measure the influence of the heavy tails of the intercell distribution, it is necessary to monitor long time intervals of traffic [2], in our case, $3 \mathrm{~s}$ of traffic at $150 \mathrm{Mb} / \mathrm{s}$.

The EDFA transient time response to input power change was calculated numerically solving a system of differential equations by using a fast Runge-Kutta method [1], calculated in 10 points per individual cell. The calculated output power on slots carrying a cell was arranged in a histogram comprising 500 bins of width $0.04 \mathrm{dBm}$, uniformly tiling the range between 1 and $21 \mathrm{dBm}$. The result was a histogram estimation of the probability density function (pdf) of the output power for each of the 16 channels. 


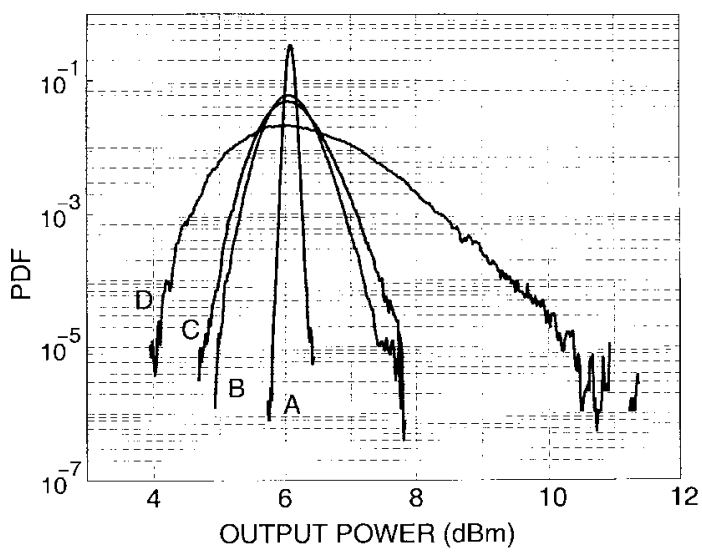

Fig. 1. Simulated pdf of output power of the channel at $\lambda=1544 \mathrm{~nm}$. Network utilization $\varrho=0.5$. Bit rate $150 \mathrm{Mb} / \mathrm{s}$. (a) $\alpha_{\text {on }}=\alpha_{\text {off }}=5.0$. (b) $\alpha_{\text {on }}=\alpha_{\text {off }}=2.1$. (c) $\alpha_{\text {on }}=\alpha_{\text {off }}=1.9$. (d) $\alpha_{\text {on }}=\alpha_{\text {off }}=1.2$.

Excited state-absorption and ion-ion cooperative upconversion are not taken into account in the model [1] used here. This model has been verified experimetally and provides a good description of transients on time scales of fractions of microseconds [1]-[4].

\section{SimUlation ResUltS}

In Fig. 1, one wavelength channel (the channel at $\lambda=1544$ nm) is shown for network utilization $\rho=0.5$ and various degrees of variability $\alpha \triangleq \alpha_{\mathrm{on}}=\alpha_{\mathrm{off}}$. The maximum for all curves occurs at the same point of roughly 6-dBm output power, corresponding to a gain of $9 \mathrm{~dB}$. This same gain is obtained at steady state with continuous-wave $(\mathrm{CW})$ channels with $P_{\text {in }}^{\prime} \triangleq \rho P_{\text {in }}=-6$-dBm input power, corresponding to the same average total input power into the EDFA. Due to the nonflat gain spectrum of the amplifier, the steady-state gain at $\lambda=1559 \mathrm{~nm}$ is $2.5 \mathrm{~dB}$ higher.

The EDFA gain has two limit points: a zero load, small signal limit when no packet is present, of value $34 \mathrm{~dB}$; and a full load limit when packets at all wavelengths are always present, of value $6 \mathrm{~dB}$. The relatively smooth traffic of curve A $(\alpha=5.0)$, results in moderate power swings; as the variability increases (curve B: $\alpha=2.1$; curve $\mathrm{C}: \alpha=1.9$ ) the power swings increase too, reaching substantial values for the "truly self-similar" case of $\alpha=1.2$ depicted in curve $\mathrm{D}$, where power swings in excess of $6 \mathrm{dBm}$ have probability $10^{-5}$. Note that power swings increase on both sides of the maximum power: high variability of ON periods means long trains of packets giving time to the EDFA gain to deeply saturate and move closer to its lower (full load) limit. Likewise, high variability of the OFF periods means long lulls during which the EDFA has no power in, giving the pump enough time to re-invert the erbium ions, and thus driving the gain on the next cell arrival toward its upper (zero load) limit.

We next investigate the influence of the network utilization, restricting our attention to the self-similar case $\alpha=1.2$. Fig. 2 depicts the histogram of the output power swings versus the output power differential (output power relative to the most probable output power level, i.e., that corresponding to the maximum level of the histogram); this is done for a fair

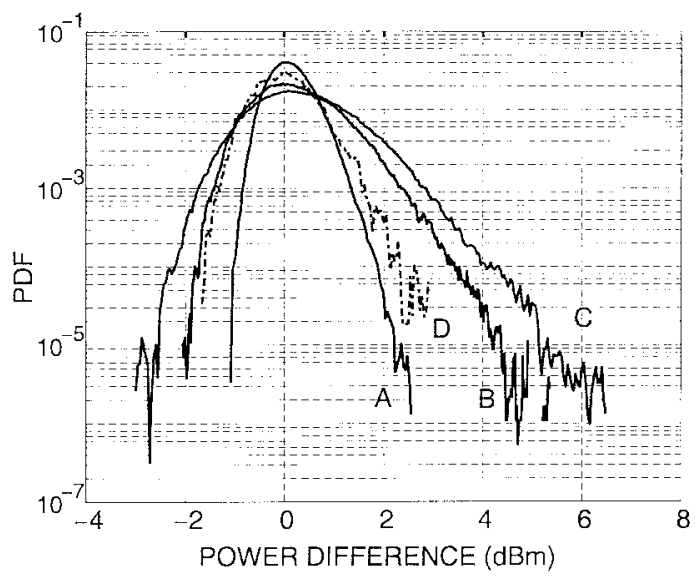

Fig. 2. Simulated pdf of the output power difference relative to the maximum probability level of the channel at $\lambda=1544 \mathrm{~nm}$. Bit rate $150 \mathrm{Mb} / \mathrm{s}$. (a) $\alpha_{\text {on }}=1.2, \alpha_{\text {off }}=1.64, \varrho=0.7$. (b) $\alpha_{\text {on }}=\alpha_{\text {off }}=1.2, \varrho=0.5$. (c) $\alpha_{\text {on }}=1.64, \alpha_{\text {off }}=1.2, \varrho=0.3$. (d) Same as (b), but with bit rate $2.5 \mathrm{~Gb} / \mathrm{s}$.

comparison, as different network utilizations result in different most-probable powers. Curve A represents a self-similar case with $\rho=0.7, \alpha_{\text {on }}=1.2$ and $\alpha_{\text {off }}=1.64$; curve B has $\rho=0.5, \alpha_{\text {on }}=\alpha_{\text {off }}=1.2$; curve $\mathrm{C}$ has $\rho=0.3, \alpha_{\text {on }}=1.64$, $\alpha_{\text {off }}=1.2$. As the network utilization increases, the power swings decrease since for small utilization there are fewer packets flowing and hence the self-similarity in the OFF periods contributes to a much more dramatic effect. Hence, there is a sizable difference between curve B $(\rho=0.5)$ and curve A $(\rho=0.7)$ for positive power differentials. For larger utilization factors, however, the ON periods, while more variable, are long enough to keep the amplifier in deep saturation, hence the curves are much closer in the negative side of the power difference. Curve A has a most-probable gain of $7.5 \mathrm{~dB}$, already very close to the lower limit, and it approaches that limit asymptotically at $-1.5 \mathrm{dBm}$ as seen in Fig. 2 .

In Fig. 2, we can also see the influence of the bit rate on the histograms. Curve D has the same variability and utilization as curve $\mathrm{B}$, but the bit rate is increased to $2.5 \mathrm{~Gb} / \mathrm{s}$. Evidently, smaller packet duration $(0.17 \mu \mathrm{s})$ gives the EDFA less time to experience large gain swings, which is reflected in output power swings of $5 \mathrm{dBm}$ (extrapolated at $10^{-5}$ ) as compared to over $6-\mathrm{dBm}$ swings for the corresponding $150-\mathrm{Mb} / \mathrm{s}$ case on curve B.

Finally, the dependence behavior of the power swings on wavelength is investigated in Fig. 3, where the histograms versus the power difference for the channel at $\lambda=1559 \mathrm{~nm}$ (curve A) and for the channel at $\lambda=1544 \mathrm{~nm}$ (curve B) are depicted. The power swings for the channel at $\lambda=1544$ $\mathrm{nm}$ are larger due to the fact that the transients are faster in this spectral region [4], hence the EDFA gain can swing more in the same time interval. In analogy to this observation, substantially larger power swings will be observed in the case of cascades of EDFA's where the transients are much faster and reach even submicrosecond levels [4].

\section{CONCLUSION}

The influence of the gain dynamics on system performance for packetized data in WDM networks was investigated. We 


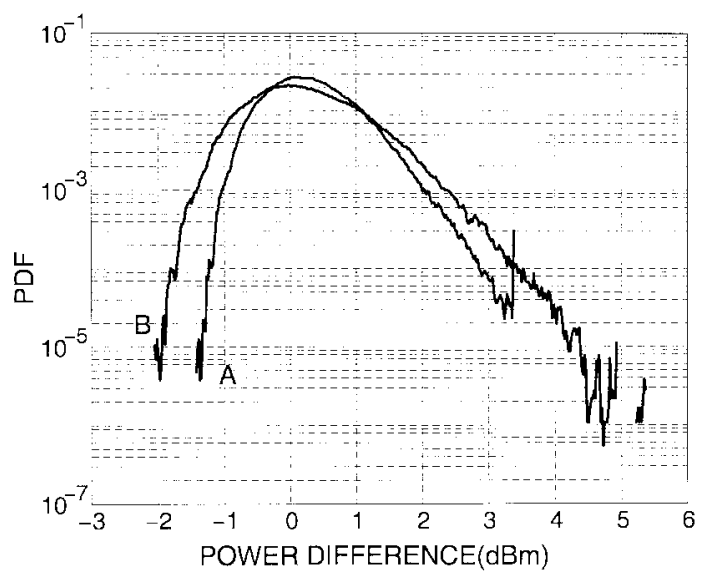

Fig. 3. Simulated pdf of the output power difference relative to the maximum probability level. Network utilization $\varrho=0.5, \alpha_{\text {on }}=\alpha_{\text {off }}=1.2$. Bit rate $150 \mathrm{Mb} / \mathrm{s}$. (a) Output of the channel at $\lambda=1559 \mathrm{~nm}$. (b) Output of the channel at $\lambda=1544 \mathrm{~nm}$.

have analyzed the power swings at the output of a single EDFA as a function of the variability of the network traffic, the network utilization factor and the spectral characteristics. The extensive simulation model built for this purpose points to large output power swings in the case of traffic with highly variable packet interarrival times, such as multimedia traffic. Power swings in excess of $6 \mathrm{dBm}$ were found in the case of self-similar traffic. Investigations indicate that the effect is much more profound when high-variability traffic is fed into cascades of amplifiers. Faster transients connected to ion-ion pairing in strongly doped fibers [5], and not captured by the fast EDFA model employed, should result in faster transients, in which case the observed swings in our simulations would be larger. This effect, however, due to the usually low concentration of ion pairs $(1 \%-5 \%)$ [5], should not considerably affect the overall EDFA time response.

\section{REFERENCES}

[1] Y. Sun, G. Luo, J. L. Zyskind, A. A. M. Saleh, A. K. Srivastava, and J. W. Sulhoff, "Model for gain dynamics in erbium-doped fiber amplifiers," Electron. Lett., vol. 32, pp. 1490-1491, Aug. 1996.

[2] W. Willinger, M. S. Taqqu, R. Sherman, and D. V. Wilson, "Selfsimilarity through high-variability: Statistical analysis of ethernet LAN traffic at the source level," IEEE/ACM Trans. Networking, vol. 5, pp. 71-86, Feb. 1997.

[3] E. Desurvire, Erbium-Doped Fiber Amplifiers. New York, Wiley, 1994.

[4] Y. Sun, A. K. Srivastava, J. L. Zyskind, J. W. Sulhoff, C. Wolf, and R. W. Tkach, "Fast power transients in WDM optical networks with cascaded EDFA's," Electron. Lett., vol. 33, pp. 313-314, Feb. 1997.

[5] P. Myslinski, D. Nguyen, and J. Chrostowski, "Effects of concentration on the performance of erbium-doped fiber amplifiers," J. Lightwave Technol., vol. 15, pp. 112-120, Jan. 1997. 